\section{ALZHEIMER DISEASE}

\section{$A P O E^{\star} \varepsilon 4$ is linked to BBB breakdown}

The $\varepsilon 4$ allele of the apolipoprotein $E$ gene $\left(\mathrm{APOE}^{\star} \varepsilon 4\right)$ is a well-established genetic risk factor for Alzheimer disease (AD), but the mechanisms through which this variant influences $A D$ risk remain unclear. As reported in Nature, a new study by Berislav Zlokovic and colleagues demonstrates an association between $A P O E^{\star} \varepsilon 4$ carrier status and bloodbrain barrier (BBB) breakdown, which could contribute to cognitive decline.

The investigators recruited 435 participants aged $\geq 45$ years, 245 of whom underwent dynamic contrast-enhanced MRI of the brain to measure BBB integrity. $A P O E^{\star} \varepsilon 4$ carriers that is, people with the $A P O E^{\star} \varepsilon 3 / \varepsilon 4$ or the $A P O E^{*} \varepsilon 4 / \varepsilon 4$ genotype - showed increased BBB breakdown in the hippocampus and parahippocampal gyrus compared with $A P O E^{*} \varepsilon 3 / \varepsilon 3$ individuals, even in the absence of cognitive impairment. BBB damage was not linked to amyloid- $\beta$ or tau accumulation in the brain, suggesting that it occurred independently of $\mathrm{AD}$ pathology.

To examine whether the APOE genotype and/or BBB dysfunction could predict future cognitive decline, the researchers used soluble platelet-derived growth factor receptor- $\beta$ (sPDGFR $\beta$ ) in the cerebrospinal fluid (CSF) as a marker of BBB damage. Among 146 participants who underwent longitudinal assessments, the combination of $A P O E^{*} \varepsilon 4$ carrier status and high levels of sPDGFR $\beta$ in the CSF at baseline was associated with accelerated cognitive decline, and with evidence of increased activity of the cyclophilin A-matrix metalloproteinase 9 (CypA-MMP9) pathway.

"This study sheds light on a new way of looking at this disease and possibly treatment in people with the $A P O E^{\star} \varepsilon 4$ gene, looking at blood vessels and improving their function to potentially slow down or arrest cognitive decline," concludes Zlokovic. The authors point out that the CypA-MMP9 pathway has previously been implicated in BBB breakdown and could represent a target for therapeutic intervention in $\mathrm{APOE}^{\star} \varepsilon 4$ carriers.

Heather Wood

ORIGINAL ARTICLE Montagne, A. et al. APOE4 leads to blood-brain barrier dysfunction predicting cognitive decline. Nature 581, 71-76 (2020)

RELATED ARTICLES Sweeney, M. D. et al. Blood-brain barrie breakdown in Alzheimer disease and other neurodegenerative disorders. Nat. Rev. Neurol. 14, 133-150 (2018) | Yamazaki, Y. et al. Apolipoprotein E and Alzheimer disease: pathobiology and targeting strategies. Nat. Rev. Neurol. 15, 501-518 (2019)

\title{
NLRP3 inflammasome activity as biomarker for primary progressive multiple sclerosis
}

Increased activity of the NLRP3 inflammasome in immune cells in the blood is a potential biomarker of primary progressive multiple sclerosis (PPMS), according to a new study published in Brain. The finding could also provide new targets for therapeutic intervention.

"Prognostic molecular biomarkers for MS are lacking", explains Manuel Comabella, who led the study alongside Sunny Malhotra. "Biomarkers are particularly needed for the progressive form of the disease, for which there is no effective therapy."

The researchers performed RNA sequencing on peripheral blood mononuclear cells and found that the expression of multiple pro-inflammatory genes, including those encoding IL- $1 \beta$ and NLRP3, was higher in participants with PPMS than in participants with other forms of MS and in healthy controls. This finding was replicated with real-time PCR in a second cohort. NLRP3 forms a protein complex called an inflammasome, which mediates secretion of IL- $1 \beta$.
During a follow-up period, participants with PPMS who had high IL-1 $\beta$ mRNA levels needed walking assistance an average of 11 years earlier than their counterparts with low IL- $1 \beta$ mRNA levels, suggesting that IL- $1 \beta$ expression is a prognostic marker. IL- $1 \beta$ expression was not associated with disease progression in participants with other forms of MS, indicating that the marker could be specific for PPMS.

The authors suggest that this finding could inform the search for new MS therapies. "At present, we are working on the identification of specific NLRP3 inflammasome inhibitors that totally suppress IL- $1 \beta$ secretion and have the potential to become effective treatments by preventing disease progression in patients with PPMS," concludes Comabella.

Sarah Lemprière

ORIGINAL ARTICLE Malhotra, S. et al. NLRP3 inflammasome as prognostic factor and therapeutic target in primary progressive multiple sclerosis patients. Brain https://doi.org/ 10.1093/brain/awaa084 (2020)

MOTOR NEURON DISEASE

\section{Does the presenting phenotype predict survival in ALS-FTD?}

In patients with both amyotrophic lateral sclerosis and frontotemporal dementia (ALS-FTD), the initial presenting symptoms can provide clues as to the future trajectory of the disease, according to new research published in Neurology.

"We wanted to investigate whether disease progression in patients with ALS-FTD differed depending on whether they present first with motor or cognitive symptoms," explains corresponding author Rebekah Ahmed. "This question has implications for prognostication and also for disentangling fast and slow progressors in clinical trials."

The study included 59 patients with ALSFTD who were recruited from clinics at the Brain and Mind Centre, University of Sydney, Australia. Among these individuals, 41 (69\%) had initially presented with cognitive symptoms and $18(31 \%)$ had initially presented with motor symptoms.

The researchers found that median survival from the time of symptom onset was considerably shorter in the patients with motor presentation than in those with cognitive presentation ( 2.7 years versus 4.4 years), despite similar levels of cognitive functioning between the groups at the beginning of the study.

"The main conclusion that can be drawn is that survival in ALS-FTD is not uniformly poor, and that it depends on whether patients develop motor or cognitive symptoms first," comments Ahmed. "The findings suggest that progression in ALS-FTD is linked to motor and physiological changes rather than to cognitive changes."

The investigators suggest that future research should focus on the pathological determinants of survival in patients with ALS-FTD, including a potential role for neurodegeneration in the motor cortex.

Heather Wood

ORIGINAL ARTICLE Ahmed, R. M. et al. Phenotypic variability in ALS-FTD and effect on survival. Neurology https://doi.org/10.1212/WNL.0000000000009398 (2020) RELATED ARTICLES Swinnen, B. \& Robberecht, W. The phenotypic variability of amyotrophic lateral sclerosis. Nat. Rev. Neurol. 10, 661-670 (2014) 